\title{
A Generalized Grothendieck Inequality and Nonlocal Correlations that Require High Entanglement
}

\author{
Jop Briët ${ }^{1, \star}$, Harry Buhrman ${ }^{1, \star}$, Ben Toner ${ }^{2, \star \star}$ \\ 1 Centrum Wiskunde \& Informatica (CWI), and University of Amsterdam, Science Park 123, \\ 1098 SJ Amsterdam, The Netherlands. E-mail: j.briet@cwi.nl; jop.briet@cwi.nl; buhrman@cwi.nl \\ 2 School of Physics, The University of Melbourne, Victoria 3010, Australia. E-mail: bentoner@ bentoner.com
}

Received: 24 December 2010 / Accepted: 18 April 2011

Published online: 13 June 2011 - (c) The Author(s) 2011. This article is published with open access at Springerlink.com

\begin{abstract}
Suppose that Alice and Bob make local two-outcome measurements on a shared entangled quantum state. We show that, for all positive integers $d$, there exist correlations that can only be reproduced if the local Hilbert-space dimension is at least $d$. This establishes that the amount of entanglement required to maximally violate a Bell inequality must depend on the number of measurement settings, not just the number of measurement outcomes. We prove this result by establishing a lower bound on a new generalization of Grothendieck's constant.
\end{abstract}

\section{Introduction}

Grothendieck's inequality first arose in the study of norms on tensor products of Banach spaces [Gro53]. It has since found many applications in mathematics and computer science, including approximation algorithms [AN04,CW04,BOFV10a] and communication complexity [LS07,LMSS08]. In quantum information, Grothendieck's inequality quantifies the difference between the classical and quantum values of certain simple Bell inequalities, as established by Tsirelson [Tsi87]. Tsirelson's work has been the starting point of considerable recent research into quantum nonlocality [CHTW04, AGT06, RT07, $\mathrm{BPA}^{+}$08, $\mathrm{PGWP}^{+}$08, BBLV09, $\mathrm{JPPG}^{+}$10].

We start by stating the inequality in its strongest form, in terms of the real Grothendieck constant $K_{G}$.

^ Supported by Vici grant 639-023-302 from the Netherlands Organization for Scientific Research (NWO), by the European Commission under the Integrated Project Qubit Applications (QAP) funded by the IST directorate as Contract Number 015848, and EU QCS grant.

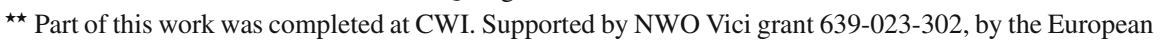
Commission under the Integrated Project QAP funded by the IST directorate as Contract Number 015848. 
Definition 1. The real Grothendieck constant of order $n$, is the smallest real number $K_{G}(n)$ such that: For all positive integers $r$ and for all real $r \times r$ matrices $M=\left(M_{i j}\right)$, the inequality

$$
\max _{\substack{\boldsymbol{a}_{1}, \ldots, \boldsymbol{a}_{r} \\ \boldsymbol{b}_{1}, \ldots, \boldsymbol{b}_{r}}} \sum_{i, j} M_{i j} \boldsymbol{a}_{i} \cdot \boldsymbol{b}_{j} \leq K_{G}(n) \max _{\substack{\alpha_{1}, \ldots, \alpha_{r} \\ \beta_{1}, \ldots, \beta_{r}}} \sum_{i, j} M_{i j} \alpha_{i} \beta_{j}
$$

holds, where the maximum on the left-hand side is taken over all sequences $\boldsymbol{a}_{1}, \ldots, \boldsymbol{a}_{r}, \boldsymbol{b}_{1}, \ldots, \boldsymbol{b}_{r}$ of n-dimensional real unit vectors, $\boldsymbol{a}_{i} \cdot \boldsymbol{b}_{j}$ denotes the Euclidean inner product of $\boldsymbol{a}_{i}$ and $\boldsymbol{b}_{j}$, and the maximum on the right-hand side is taken over all sequences $\alpha_{1}, \ldots, \alpha_{r}, \beta_{1}, \ldots, \beta_{r}$ of real numbers in the set $\{-1,+1\}$.

The real Grothendieck constant, denoted $K_{G}$, is defined as $\lim _{n \rightarrow \infty} K_{G}(n)$.

The exact value of $K_{G}$ is unknown. The tightest version of the inequality known is due to Krivine [Kri79], who proved that $K_{G} \leq \pi /(2 \ln (1+\sqrt{2})) \approx 1.78$. Davie [Dav84] and, independently, Reeds [Ree91] are responsible for the best lower bounds: they showed that $K_{G} \gtrsim 1.68$. Raghavendra and Steurer have shown that $K_{G}$ can be approximated within an error $\eta$ in time $\exp \left(\exp \left(O\left(1 / \eta^{3}\right)\right)\right)$ [RS09].

In this paper, we give a new generalization of Grothendieck's inequality. We replace the maximization over scalars on the right-hand side of Eq. (1) with a maximization over real unit vectors of dimension $m<n$. More formally:

Definition 2. Let $m$ and $n$ be positive integers with $m<n$. Define $K_{G}(n \mapsto m)$ to be the smallest real number such that: For all positive integers $r$ and for all real $r \times r$ matrices $M=\left(M_{i j}\right)$, the inequality

$$
\max _{\substack{\boldsymbol{a}_{1}, \ldots, \boldsymbol{a}_{r} \\ \boldsymbol{b}_{1}, \ldots, \boldsymbol{b}_{r}}} \sum_{i, j} M_{i j} \boldsymbol{a}_{i} \cdot \boldsymbol{b}_{j} \leq K_{G}(n \mapsto m) \max _{\substack{\boldsymbol{a}_{1}^{\prime}, \ldots, \boldsymbol{a}_{r}^{\prime} \\ \boldsymbol{b}_{1}^{\prime}, \ldots, \boldsymbol{b}_{r}^{\prime}}} \sum_{i, j} M_{i j} \boldsymbol{a}_{i}^{\prime} \cdot \boldsymbol{b}_{j}^{\prime}
$$

holds, where the maximum on the left-hand side is taken over all sequences $\boldsymbol{a}_{1}, \ldots, \boldsymbol{a}_{r}, \boldsymbol{b}_{1}, \ldots, \boldsymbol{b}_{r}$ of $n$-dimensional real unit vectors, and the maximum on the right-hand side is taken over all sequences $\boldsymbol{a}_{1}^{\prime}, \ldots, \boldsymbol{a}_{r}^{\prime}, \boldsymbol{b}_{1}^{\prime}, \ldots, \boldsymbol{b}_{r}^{\prime}$ of $m$-dimensional real unit vectors. This generalizes Definition 1 in the sense that $K_{G}(n)=K_{G}(n \mapsto 1)$.

Building on the techniques Grothendieck himself [Gro53] used to prove the original lower bound on $K_{G}$, we prove the following lower bound on $K_{G}(n \mapsto m)$.

Theorem 1. For all $m<n$,

$$
\begin{aligned}
K_{G}(n \mapsto m) & \geq \frac{m}{n}\left(\frac{\Gamma\left(\frac{m}{2}\right)}{\Gamma\left(\frac{m+1}{2}\right)} \frac{\Gamma\left(\frac{n+1}{2}\right)}{\Gamma\left(\frac{n}{2}\right)}\right)^{2} \\
& =1+\frac{1}{2 m}-\frac{1}{2 n}-O\left(\frac{1}{m^{2}}\right) .
\end{aligned}
$$

After the appearance of this work in preprint form, it was shown in [BOFV10b] that for positive semidefinite matrices $M$, like the ones considered here, the lower bound on $K_{G}(\infty \mapsto m)$ given in Theorem 1 is in fact optimal. Note that for general matrices, trivially, we have $K_{G}(n \mapsto m) \leq K_{G}(n)$. Better upper bounds on $K_{G}(\infty \mapsto m)$ for general matrices were found in [BOFV10a]. 
Related work. Definition 2 is but the latest in a long history of generalizations of Grothendieck's inequality. Previously, Grothendieck's inequality has been generalized as follows:

- Replacing the real scalars, vectors and matrices with complex ones results in the definition of the complex Grothendieck constant.

- Restricting to positive definite matrices $M$ results in a tighter inequality [Rie74, Nes98].

- Rather than proving inequalities that hold for all matrices, we can prove inequalities that only hold for all matrices $M$ of some fixed size, say $r \times s$. This refinement has been studied by Fishburn and Reeds [FR94], and results in the definition of a constant which they denote $K_{G}(r, s)$, not to be confused with our $K_{G}(n \mapsto m)$.

- Observe that Eq. (1) has a bipartite structure, in the following sense: on the lefthand side, the sum is of inner products $\boldsymbol{a}_{i} \cdot \boldsymbol{b}_{j}$ of a vector from $\left\{\boldsymbol{a}_{1}, \ldots, \boldsymbol{a}_{r}\right\}$ with a vector from $\left\{\boldsymbol{b}_{1}, \ldots, \boldsymbol{b}_{r}\right\}$; there are no inner products $\boldsymbol{a}_{i} \cdot \boldsymbol{a}_{j}$ or $\boldsymbol{b}_{i} \cdot \boldsymbol{b}_{j}$. A similar observation applies to the right-hand side. So if we consider a graph with vertices labelled by the vectors $\boldsymbol{a}_{i}$ and $\boldsymbol{b}_{j}$, and draw an edge between vertex $\boldsymbol{a}_{i}$ and $\boldsymbol{b}_{j}$ whenever $M_{i j} \neq 0$, then the resulting "interaction graph" is bipartite. Alon et al. have generalized Grothendieck's inequality to general graphs that are not necessarily bipartite [AMMN06].

Application to quantum correlations. Suppose that Alice and Bob share an entangled quantum state and that each performs a local two-outcome measurement on their part of that state. We are interested in the (classical) correlations between their measurement outcomes, i.e., the probability that they obtain the same measurement outcome, for various choices of the local measurements. It is a well-established fact (both theoretically [Bel64,CHSH69] and experimentally [AGR81,AGR82,ADR82, TBZG98, RKM ${ }^{+}$01]), that there are entangled states for which these correlations are nonlocal, meaning that they cannot be explained in a classical universe (more formally, they are inconsistent with all local hidden variable models).

As a corollary of Theorem 1, we show that there are nonlocal quantum correlations that require entangled states with local support on a Hilbert space of dimension at least $d$, for any $d$ (even with arbitrary shared randomness). This confirms a conjecture of Brunner et al. $\left[\mathrm{BPA}^{+} 08\right]$, proving that what they term dimension witnesses exist with binary outcomes. In particular, Brunner et al. conjectured that two-outcome measurements may be sufficient to test the dimension of any bipartite quantum system. This work establishes that their conjecture is true. This strengthens the fundamental result described in the preceding paragraph: the fact that there exist nonlocal correlations is the $d=1$ case of our result.

Brunner et al. pointed out that the same result would follow if one could prove that the Grothendieck constants $K_{G}(n)$ are strictly increasing in $n$. This is plausible but we do not know how to prove it. Our proof sidesteps this issue.

In addition to the work of Brunner et al., there is some other work on lower-bounding the amount of entanglement required to reproduce certain correlations. Pál and Vértesi construct correlations that cannot be reproduced if each party has only a smalldimensional quantum system [PV08,PV09]. Wehner, Christandl and Doherty show how to obtain lower bounds using information-theoretic arguments [WCD08].

Another lower bound follows from communication complexity, but for the more general problem of reproducing correlations of measurements with more than two outcomes. The Hidden Matching quantum communication complexity problem $(\operatorname{HM}(n))$ 
[BYJK08] can be formulated as a nonlocal correlation, where a maximally entangled state of dimension $n$ is used to reproduce the correlations perfectly. On the other hand, using the classical bounded error one-way communication complexity lower bound for $\operatorname{HM}(n)$, it follows that one needs $\omega(\sqrt{n})$ bits of one-way communication to approximately reproduce these correlations classically. This in turn yields a lower bound on the dimension of the entangled state of $\sqrt{n} / \log n$ for any quantum strategy that approximates these correlations. This follows because any smaller dimensional state can be used to establish a classical one-way protocol that approximates these correlations and uses less than $\omega(\sqrt{n})$ bits of communication, by simply communicating a classical description of an approximation of the state that Bob has after Alice did her measurement. (See also [BRSW10].)

Outline. The paper is structured as follows. We define notation in Sect. 2. In Sect. 3, we rework the definition of $K_{G}(n \mapsto m)$ in order to work in the limit $r \rightarrow \infty$, which makes things simpler. Then, in Sect. 4, we prove our main result, Theorem 1. In Sect. 5, we describe the consequences for quantum nonlocality. Readers wishing to skip the details of the proof can read Sect. 5 immediately after Sect. 3.

Note. After a preliminary version of this paper was submitted to the twelfth workshop on Quantum Information Processesing (QIP 2009) on 20 October, 2008, we learned of a paper by Vértesi and Pál [VP09], who obtain similar results independently. Without explicitly defining $K_{G}(n \mapsto m)$, they prove that this quantity is strictly increasing with $m$ when $n \rightarrow \infty$ using essentially the same methods that we do, and use this result to confirm Brunner et al.'s conjecture, giving a Bell inequality that has an infinite number of measurement settings; they obtain dimension witnesses with finite measurement settings using different methods. In this paper, we obtain dimension witnesses with a finite number of measurement settings from our lower bounds on $K_{G}(n \mapsto m)$.

\section{Notation}

We write $[n]$ for the set $\{1, \ldots, n\}$. The unit sphere in $\mathbb{R}^{n}$ is denoted $S_{n-1}$. We write $\boldsymbol{d} \boldsymbol{a}$ for the Haar measure on $S_{n-1}$, normalized such that $\int \boldsymbol{d} \boldsymbol{a}=1$. The norm $\|\boldsymbol{a}\|$ of a vector $\boldsymbol{a}$ is always the Euclidean norm. In the Introduction and Sect. 6, subscripts label vectors; in the remainder of the paper, subscripts on a vector denote its components. Variables in lowercase roman type will typically be vectors on the unit sphere; variables in lowercase Greek type will typically be scalars.

\section{An Equivalent Definition of $K_{G}(n \mapsto m)$}

To establish a lower bound on $K_{G}(n \mapsto m)$ per Eq. (2), we need to exhibit an $r \times r$ matrix $M$ and then calculate (or at least bound) both sides of Eq. (2). We will work in the limit $r \rightarrow \infty$ and so we start by showing that this indeed allows us to lower bound $K_{G}(n \mapsto m)$.

Lemma 1. The constant $K_{G}(n \mapsto m)$ is bounded by

$$
K_{G}(n \mapsto m) \geq \sup _{M: S_{n-1} \times S_{n-1} \rightarrow[-1,1]}\left(\frac{1}{D(M)} \int \operatorname{dad} \boldsymbol{b} M(\boldsymbol{a}, \boldsymbol{b}) \boldsymbol{a} \cdot \boldsymbol{b}\right),
$$


where the supremum is over measurable functions $M: S_{n-1} \times S_{n-1} \rightarrow \mathbb{R}$ with $\int d \boldsymbol{a} d \boldsymbol{b}|M(\boldsymbol{a}, \boldsymbol{b})|=1$ and the denominator

$$
D(M)=\max _{A, B: S_{n-1} \rightarrow S_{m-1}} \int \boldsymbol{d a d b M}(\boldsymbol{a}, \boldsymbol{b}) \boldsymbol{A}(\boldsymbol{a}) \cdot \boldsymbol{B}(\boldsymbol{b}),
$$

with the maximum over functions $A, B: S_{n-1} \rightarrow S_{m-1}$.

Observe that we can rewrite the conventional definition of $K_{G}(n \mapsto m)$ as

$$
K_{G}(n \mapsto m)=\lim _{r \rightarrow \infty} \sup _{M_{i j}}\left(\frac{\max _{\left(\boldsymbol{a}_{i}, \boldsymbol{b}_{j}\right)} \sum_{i, j} M_{i j} \boldsymbol{a}_{i} \cdot \boldsymbol{b}_{j}}{\max _{\left(\boldsymbol{a}_{i}^{\prime}, \boldsymbol{b}_{j}^{\prime}\right)} \sum_{i, j} M_{i j} \boldsymbol{a}_{i}^{\prime} \cdot \boldsymbol{b}_{j}^{\prime}}\right)
$$

The following lemma implies Lemma 1.

Lemma 2. For any measurable function $M^{\prime}(\boldsymbol{a}, \boldsymbol{b})$ with $\boldsymbol{a}, \boldsymbol{b} \in S_{n-1}$, and any $\eta>0$, there exist an $r$ and matrix $M_{i j} \in \mathbb{R}^{r} \times \mathbb{R}^{r}$, such that

$$
\frac{\max _{\left(\boldsymbol{a}_{i}, \boldsymbol{b}_{j}\right)} \sum_{i, j} M_{i j} \boldsymbol{a}_{i} \cdot \boldsymbol{b}_{j}}{\max _{\left(\boldsymbol{a}_{i}^{\prime}, \boldsymbol{b}_{j}^{\prime}\right)} \sum_{i, j} M_{i j} \boldsymbol{a}_{i}^{\prime} \cdot \boldsymbol{b}_{j}^{\prime}} \geq \frac{\int \boldsymbol{d} \boldsymbol{a} \boldsymbol{d} \boldsymbol{b} M^{\prime}(\boldsymbol{a}, \boldsymbol{b}) \boldsymbol{a} \cdot \boldsymbol{b}}{\max _{A, B: S_{n-1} \rightarrow S_{m-1}} \int \boldsymbol{d} \boldsymbol{a} \boldsymbol{d} \boldsymbol{b} M^{\prime}(\boldsymbol{a}, \boldsymbol{b}) A(\boldsymbol{a}) \cdot B(\boldsymbol{b})}-\eta .
$$

We prove Lemma 2 using an $\varepsilon$-net, which is defined as follows.

Definition 3 ( $\varepsilon$-net). For fixed $\varepsilon>0$, a set of vectors $E_{n}^{\varepsilon}=\left\{\boldsymbol{w}_{1}, \boldsymbol{w}_{2}, \cdots \in S_{n-1}\right\}$ is an $\varepsilon$-net for $S_{n-1}$ iffor all $\boldsymbol{a} \in S_{n-1}$, there exists a vector $\boldsymbol{u} \in E$ that satisfies $\|\boldsymbol{a}-\boldsymbol{u}\|_{2} \leq \varepsilon$.

Lemma 3. For $0<\varepsilon<1$, there is an $\varepsilon$-net for $S_{n-1}$ with $\left|E_{n}^{\varepsilon}\right|=(3 / \varepsilon)^{n}$.

Proof. We follow a standard volume argument (see e.g. [Pis99, Lemma 4.10]). Let $E_{n}^{\varepsilon}$ be a maximal set of vectors satisfying $\|\boldsymbol{u}-\boldsymbol{v}\|_{2} \geq \varepsilon$ for all $\boldsymbol{u}, \boldsymbol{v} \in E_{n}^{\varepsilon}$, where the existence of such a set is guaranteed by Zorn's Lemma. Then $E_{n}^{\varepsilon}$ is an $\varepsilon$-net for $S_{n-1}$. We bound $\left|E_{n}^{\varepsilon}\right|$ using a volume argument. The open balls of radius $\varepsilon / 2$ around each point $u \in E_{n}^{\varepsilon}$ are pairwise disjoint and all contained in the ball of radius $1+\varepsilon / 2$ about the origin. Hence

$$
\left|E_{n}^{\varepsilon}\right| \leq \frac{(1+\varepsilon / 2)^{n}}{(\varepsilon / 2)^{n}}=\left(\frac{2}{\epsilon}+1\right)^{n} \leq\left(\frac{3}{\varepsilon}\right)^{n}
$$

Proof (of Lemma 2). For some $\varepsilon>0$ let $E_{n}^{\varepsilon}=\left\{\boldsymbol{w}_{1}, \boldsymbol{w}_{2}, \ldots\right\} \subseteq S_{n-1}$ be an $\varepsilon$-net for $S_{n-1}$. Suppose we use this net, to divide the unit $n$-sphere up into $\left|E_{n}^{\varepsilon}\right|$ disjoint regions: For $w \in E_{n}^{\varepsilon}$, let $R_{w}$ be the set of points on $S_{n-1}$ that are closer to $w$ than to any other point in $E_{n}^{\varepsilon}$, and assign points equidistant to two or more points in the net in some arbitrary way. We set

$$
M_{i j}=\int_{\boldsymbol{a} \in R_{\boldsymbol{w}_{i}}} \int_{\boldsymbol{b} \in R_{\boldsymbol{w}_{j}}} \operatorname{dadb} M^{\prime}(\boldsymbol{a}, \boldsymbol{b}) .
$$


Define $\delta:=\max _{i, j}\left\{\left|\boldsymbol{w}_{i} \cdot \boldsymbol{w}_{j}-\boldsymbol{a} \cdot \boldsymbol{b}\right| \mid \boldsymbol{a} \in R_{\boldsymbol{w}_{i}}, \boldsymbol{b} \in R_{\boldsymbol{w}_{j}}\right\}$. Then by the triangle inequality, we have

$$
\begin{aligned}
& \left|\sum_{i, j} \int_{\boldsymbol{a} \in R_{w_{i}}} \boldsymbol{d a} \int_{\boldsymbol{b} \in R_{w_{j}}} d \boldsymbol{b} M(\boldsymbol{a}, \boldsymbol{b})\left(\boldsymbol{w}_{i} \cdot \boldsymbol{w}_{j}-\boldsymbol{a} \cdot \boldsymbol{b}\right)\right| \\
& \leq \sum_{i, j} \int_{\boldsymbol{a} \in R_{w_{i}}} \boldsymbol{d a} \int_{\boldsymbol{b} \in R_{w_{j}}} \boldsymbol{d b}\left|M^{\prime}(\boldsymbol{a}, \boldsymbol{b})\right|\left|\boldsymbol{w}_{i} \cdot \boldsymbol{w}_{j}-\boldsymbol{a} \cdot \boldsymbol{b}\right| \leq \delta .
\end{aligned}
$$

Hence, for the numerators we get:

$$
\max _{\left(\boldsymbol{a}_{i}, \boldsymbol{b}_{j}\right)} \sum_{i, j} M_{i j} \boldsymbol{a}_{i} \cdot \boldsymbol{b}_{j} \geq \int \boldsymbol{d a d} \boldsymbol{d} M^{\prime}(\boldsymbol{a}, \boldsymbol{b}) \boldsymbol{a} \cdot \boldsymbol{b}-\delta .
$$

For the denominators, we have

$$
\max _{A, B: S_{n-1} \rightarrow S_{m-1}} \int \boldsymbol{d} \boldsymbol{a} \boldsymbol{d} \boldsymbol{b} M^{\prime}(\boldsymbol{a}, \boldsymbol{b}) A(\boldsymbol{a}) \cdot B(\boldsymbol{b}) \geq \max _{\left(\boldsymbol{a}_{i}^{\prime}, \boldsymbol{b}_{j}^{\prime}\right)} \sum_{i, j} M_{i j} \boldsymbol{a}_{i}^{\prime} \cdot \boldsymbol{b}_{j}^{\prime},
$$

since we can always pick $A(\boldsymbol{a})=\boldsymbol{a}_{i}^{\prime}$ and $B(\boldsymbol{b})=\boldsymbol{b}_{j}^{\prime}$ for all $\boldsymbol{a} \in R_{i}$ and $\boldsymbol{b} \in R_{j}$. The result follows from the fact that we can let $\delta$ become arbitrarily small by decreasing $\varepsilon$. To see this, note that by the Cauchy-Schwarz inequality, we have for every $i, j \in\left\{1, \ldots,\left|E_{n}^{\varepsilon}\right|\right\}$ and $\boldsymbol{a} \in R_{\boldsymbol{w}_{i}}$ and $\boldsymbol{b} \in R_{\boldsymbol{w}_{j}}$,

$$
\begin{aligned}
\boldsymbol{w}_{i} \cdot \boldsymbol{w}_{j}-\boldsymbol{a} \cdot \boldsymbol{b} & =\left(\boldsymbol{w}_{i}-\boldsymbol{a}\right) \cdot \boldsymbol{w}_{j}+\left(\boldsymbol{w}_{j}-\boldsymbol{b}\right) \cdot \boldsymbol{a} \\
& \leq\left\|\boldsymbol{w}_{i}-\boldsymbol{a}\right\|_{2}+\left\|\boldsymbol{w}_{j}-\boldsymbol{b}\right\|_{2} \\
& \leq 2 \varepsilon .
\end{aligned}
$$

Hence, we have $\delta \leq 2 \varepsilon$.

Although the inequality in Eq. (5) is sufficient for our need, it is not hard to see that in fact equality holds. We give a formal proof of this fact in Sect. 6.

\section{Lower Bound on $K_{G}(n \mapsto m)$}

We prove Theorem 1 by considering a specific example due to Grothendieck himself [Gro53]: For $\boldsymbol{a}, \boldsymbol{b} \in S_{n-1}$, take $M(\boldsymbol{a}, \boldsymbol{b})=\boldsymbol{a} \cdot \boldsymbol{b}$.

We start by calculating the denominator $D(M)$. To do this, we need to work out which embeddings $A, B: S_{n-1} \rightarrow S_{m-1}$ achieve the maximum in Eq. (6). It turns out that the maximum is achieved when $A$ and $B$ are equal. Informally, we should try to preserve as much of the structure of $S_{n-1}$ as possible, and it is natural to conjecture that the best embedding is a projection onto an $m$-dimensional subspace. This is indeed the case. We prove this in the following lemma.

Lemma 4. For the function $M(\boldsymbol{a}, \boldsymbol{b})=\boldsymbol{a} \cdot \boldsymbol{b}$, the optimal embedding $A: S_{n-1} \rightarrow S_{m-1}$ is a projection. In particular, the denominator $D(M)$ is given by

$$
D=\frac{1}{m}\left(\int \boldsymbol{d} \boldsymbol{a}\left(\sum_{i=1}^{m} a_{i}^{2}\right)^{1 / 2}\right)^{2}
$$

where $a_{1}, \ldots, a_{n}$ are the components of $\boldsymbol{a}$. 
Proof. We prove this result in two steps. First, we show that the maximum is achieved by a weighted projection. Second, we show that the best projection is the one with uniform weights.

We need to calculate

$$
D(M)=\max _{A, B: S_{n-1} \rightarrow S_{m-1}} \int \operatorname{dadbM}(\boldsymbol{a}, \boldsymbol{b}) \boldsymbol{A}(\boldsymbol{a}) \cdot \boldsymbol{B}(\boldsymbol{b}),
$$

with the maximum over functions $A, B: S_{n-1} \rightarrow S_{m-1}$. For $M(\boldsymbol{a}, \boldsymbol{b})=\boldsymbol{a} \cdot \boldsymbol{b}$, we can write

$$
(\boldsymbol{a} \cdot \boldsymbol{b})(A(\boldsymbol{a}) \cdot B(\boldsymbol{b}))=(\boldsymbol{a} \otimes A(\boldsymbol{a})) \cdot(\boldsymbol{b} \otimes B(\boldsymbol{b})),
$$

(this trick is motivated by a similar one used by Krivine in proving his upper bound on $K_{G}$ [Kri79]), which allows us to write $D(M)$ as a maximization over the inner product of two vectors,

$$
\begin{aligned}
D & =\max _{A, B: S_{n-1} \rightarrow S_{m-1}}\left(\int \boldsymbol{d} \boldsymbol{a} \boldsymbol{a} \otimes A(\boldsymbol{a})\right) \cdot\left(\int \boldsymbol{d} \boldsymbol{b} \boldsymbol{b} \otimes B(\boldsymbol{b})\right) \\
& =\max _{A: S_{n-1} \rightarrow S_{m-1}}\left\|\int \boldsymbol{d} \boldsymbol{a} \boldsymbol{a} \otimes A(\boldsymbol{a})\right\|^{2},
\end{aligned}
$$

where the second equality follows from the fact that the inner product is maximized when vectors are parallel. Let $\int \boldsymbol{d} \boldsymbol{a} \boldsymbol{a} \otimes A(\boldsymbol{a})=\chi \boldsymbol{v}$, where $\boldsymbol{v}$ is an $(n+m)$-dimensional unit vector and $\chi \geq 0$ is what we want to maximize. Applying the singular value decomposition - known in quantum information theory as the Schmidt decomposition (see, for example [NC00])—we can write

$$
\boldsymbol{v}=\sum_{i=1}^{m} \sqrt{\gamma_{i}} \boldsymbol{x}_{i} \otimes \boldsymbol{y}_{i},
$$

where, for each $i \in[m], \gamma_{i} \geq 0, \sum_{i} \gamma_{i}=1$, and $\left\{\boldsymbol{x}_{1}, \ldots, \boldsymbol{x}_{m}\right\}$ and $\left\{\boldsymbol{y}_{1}, \ldots, \boldsymbol{y}_{m}\right\}$ are orthonormal sets in $\mathbb{R}^{n}$ and $\mathbb{R}^{m}$ respectively. Therefore, in order to maximize

$$
\begin{aligned}
\chi & =\boldsymbol{v} \cdot \int \boldsymbol{d} \boldsymbol{a} \boldsymbol{a} \otimes A(\boldsymbol{a}) \\
& =\int \boldsymbol{d} \boldsymbol{a} \sum_{i} \sqrt{\gamma_{i}}\left(\boldsymbol{a} \cdot \boldsymbol{x}_{i}\right)\left(A(\boldsymbol{a}) \cdot \boldsymbol{y}_{i}\right) \\
& =\int \boldsymbol{d} \boldsymbol{a} A(\boldsymbol{a}) \cdot\left(\sum_{i} \sqrt{\gamma_{i}}\left(\boldsymbol{a} \cdot \boldsymbol{x}_{i}\right) \boldsymbol{y}_{i}\right),
\end{aligned}
$$

we should choose $A(\boldsymbol{a})$ to be

$$
\frac{\sum_{i} \sqrt{\gamma_{i}}\left(\boldsymbol{a} \cdot \boldsymbol{x}_{i}\right) \boldsymbol{y}_{i}}{\left\|\sum_{i} \sqrt{\gamma_{i}}\left(\boldsymbol{a} \cdot \boldsymbol{x}_{i}\right) \boldsymbol{y}_{i}\right\|}=\frac{\sum_{i} \sqrt{\gamma_{i}}\left(\boldsymbol{a} \cdot \boldsymbol{x}_{i}\right) \boldsymbol{y}_{i}}{\left(\sum_{i} \gamma_{i}\left(\boldsymbol{a} \cdot \boldsymbol{x}_{i}\right)^{2}\right)^{1 / 2}},
$$

a weighted projection onto some $m$-dimensional subspace, the particular choice of which does not matter. Substituting this into Eq. (15) and then Eq. (13) and choosing a basis for $\mathbb{R}^{n}$ by extending $\boldsymbol{x}_{1}, \ldots, \boldsymbol{x}_{m}$ so that $a_{i}=\boldsymbol{a} \cdot \boldsymbol{x}_{i}$ establishes that

$$
D=\max _{A, B: S_{n-1} \rightarrow S_{m-1}} \int \boldsymbol{d a d} \boldsymbol{b}(\boldsymbol{a} \cdot \boldsymbol{b})(A(\boldsymbol{a}) \cdot B(\boldsymbol{b}))=\left(\chi\left(\gamma_{1}, \ldots, \gamma_{m}\right)\right)^{2},
$$


where

$$
\chi\left(\gamma_{1}, \ldots, \gamma_{m}\right)=\int \boldsymbol{d} \boldsymbol{a}\left(\sum_{i=1}^{m} \gamma_{i} a_{i}^{2}\right)^{1 / 2}
$$

It remains to show that weights $\gamma_{i}$ can be taken to be equal. To prove this, suppose that $\chi$ is maximized by $\left(\gamma_{1}^{*}, \gamma_{2}^{*}, \ldots, \gamma_{m}^{*}\right)$. Then, by symmetry, the maximum is also achieved by $\left(\gamma_{2}^{*}, \gamma_{1}^{*}, \ldots, \gamma_{m}^{*}\right)$, and indeed, by any other permutation $\sigma$ of the $\gamma_{i}^{*}$. Hence

$$
\begin{aligned}
\chi\left(\gamma_{1}^{*}, \ldots, \gamma_{m}^{*}\right) & =\frac{1}{m !} \sum_{\sigma} \chi\left(\gamma_{\sigma(1)}^{*}, \ldots, \gamma_{\sigma(m)}^{*}\right) \\
& =\frac{1}{m !} \sum_{\sigma} \int d a\left(\sum_{i=1}^{m} \gamma_{\sigma(i)} a_{i}^{2}\right)^{1 / 2} \\
& =\int d a \frac{1}{m !} \sum_{\sigma}\left(\sum_{i=1}^{m} \gamma_{\sigma(i)}^{*} a_{i}^{2}\right)^{1 / 2} \\
& \leq \int d a\left(\frac{1}{m !} \sum_{\sigma} \sum_{i=1}^{m} \gamma_{\sigma(i)}^{*} a_{i}^{2}\right)^{1 / 2}
\end{aligned}
$$

by Jensen's inequality and the concavity of $(\cdot)^{1 / 2}$. But the coefficient of $a_{i}^{2}$ in this expression is just

$$
\frac{1}{m !} \sum_{\sigma} \gamma_{\sigma(i)}^{*}=\frac{1}{m} \sum_{i} \gamma_{i}^{*}=\frac{1}{m} \times 1=\frac{1}{m} .
$$

Thus the maximum is achieved by uniform weights.

With Lemma 4 in hand, the proof of Theorem 1 is straightforward.

Proof (of Theorem 1). Take $M(\boldsymbol{a}, \boldsymbol{b})=\boldsymbol{a} \cdot \boldsymbol{b}$ in Lemma 5. It follows from Lemma 4 that

$$
K_{G}(n \mapsto m) \geq \frac{m}{n}\left(\frac{Y_{n}}{Y_{m}}\right)^{2},
$$

where

$$
Y_{k}:=\int_{\boldsymbol{a} \in S_{n-1}} \boldsymbol{d} \boldsymbol{a}\left(\sum_{i=1}^{k} a_{i}^{2}\right)^{1 / 2},
$$

and we evaluated the numerator in Eq. (5) by observing that it is the same as the denominator when $m=n$, and so we already calculated it as a special case of Lemma 4. We can evaluate $Y_{k}$ using a trick similar to that used to calculate the surface area of the $n$-sphere. Define

$$
C_{k}:=\int_{\boldsymbol{a} \in \mathbb{R}^{n}} \boldsymbol{d a}\left(\sum_{i=1}^{k} a_{i}^{2}\right)^{1 / 2} e^{-\|\boldsymbol{a}\|_{2}^{2}} .
$$


Introducing spherical coordinates, and writing $r=\|\boldsymbol{a}\|_{2}$, we have

$$
C_{k}=Y_{k} \Omega_{n} \int_{0}^{\infty} d r r^{n-1}\left(r^{2}\right)^{1 / 2} e^{-r^{2}}=Y_{k} \pi^{n / 2} \frac{\Gamma\left(\frac{n+1}{2}\right)}{\Gamma\left(\frac{n}{2}\right)},
$$

where $\Gamma$ is the well-known gamma function, and we pick up a factor of $\Omega_{n}=$ $2 \pi^{n / 2} / \Gamma(n / 2)$, the surface area of the unit sphere in $n$ dimensions, because of our normalization convention.

On the other hand, we have

$$
C_{k}=\int_{-\infty}^{\infty} d a_{1} \cdots d a_{k}\left(\sum_{i=1}^{k} a_{i}^{2}\right)^{1 / 2} e^{-\left(a_{1}^{2}+\cdots+a_{k}^{2}\right)} \int_{-\infty}^{\infty} d a_{k+1} \cdots d a_{n} e^{-\left(a_{k+1}^{2}+\cdots+a_{n}\right)^{2}} .
$$

We can interpret $\left(\sum_{i=1}^{k} a_{i}^{2}\right)^{1 / 2}$ as the norm of a point in $k$-dimensional space, and write the first integral (over $k$ variables) as

$$
\Omega_{k} \int_{0}^{\infty} d r^{\prime} r^{\prime k} e^{-r^{\prime 2}}=\frac{2 \pi^{k / 2}}{\Gamma\left(\frac{k}{2}\right)} \cdot \frac{\Gamma\left(\frac{k+1}{2}\right)}{2} .
$$

The second integral of Eq. (26) is simply $(\sqrt{\pi})^{n-k}$. Comparing these two ways to evaluate $C_{k}$, we conclude that

$$
Y_{k}=\frac{\Gamma\left(\frac{k+1}{2}\right)}{\Gamma\left(\frac{k}{2}\right)} \cdot \frac{\Gamma\left(\frac{n}{2}\right)}{\Gamma\left(\frac{n+1}{2}\right)}
$$

and

$$
\begin{aligned}
K_{G}(n \mapsto m) & \geq \frac{m}{n}\left(\frac{Y_{n}}{Y_{m}}\right)^{2} \\
& =\frac{m}{n}\left(\frac{\Gamma\left(\frac{m}{2}\right)}{\Gamma\left(\frac{m+1}{2}\right)} \frac{\Gamma\left(\frac{n+1}{2}\right)}{\Gamma\left(\frac{n}{2}\right)}\right)^{2} .
\end{aligned}
$$

For all integers $1 \leq m<n$, this bound is nontrivial, i.e., is strictly greater than 1 . This is because the function

$$
f(n)=\frac{1}{\sqrt{n}} \frac{\Gamma\left(\frac{n+1}{2}\right)}{\Gamma\left(\frac{n}{2}\right)}
$$

is strictly increasing for $n=1,2, \ldots$ (see Sect. 7 for a proof). Asymptotically, we have

$$
K_{G}(n \mapsto m) \geq 1+\frac{1}{2 m}-\frac{1}{2 n}-O\left(\frac{1}{m^{2}}\right),
$$

where the approximation follows from the asymptotic series (see the answer to Exercise 9.60 in [GKP94])

$$
\frac{\Gamma\left(k+\frac{1}{2}\right)}{\Gamma(k)}=\sqrt{k}\left(1-\frac{1}{8 k}+\frac{1}{128 k^{2}}+\cdots\right) .
$$




\section{Quantum Nonlocality}

Here we describe the application to quantum nonlocality. Suppose that two parties, Alice and Bob, each have a $d$-dimensional quantum system, described by Hilbert spaces $\mathcal{H}_{A} \cong \mathbb{C}^{d}$ and $\mathcal{H}_{B} \cong \mathbb{C}^{d}$, respectively. Alice and Bob each make a two-outcome measurement on their own system, resulting in outcomes $\alpha, \beta \in\{ \pm 1\}$, respectively. Suppose the set of Alice's possible measurements is $M_{A}$, and the set of Bob's possible measurements is $M_{B}$. An observable is a Hermitian operator with eigenvalues in $\{ \pm 1\}$. Alice's $a^{\text {th }}$ possible measurement is specified by an observable $A_{a}$ on $\mathcal{H}_{A}$; Bob's $b^{\text {th }}$ measurement by an observable $B_{b}$ on $\mathcal{H}_{B}$ (and all observables specify valid measurements). If the joint system of Alice and Bob is in pure state $|\psi\rangle \in \mathbb{C}^{d} \otimes \mathbb{C}^{d}$, then the joint correlation - the expectation of the product of Alice and Bob's outcomes, given that Alice performs measurement $a$ and Bob measurement $b$-is

$$
E[\alpha \beta \mid a b]=\left\langle\psi\left|A_{a} \otimes B_{b}\right| \psi\right\rangle .
$$

In computer science, such correlations are studied in the context of XOR nonlocal games [CHTW04,CSUU08].

We say that a set of joint correlations, $\left\{E[\alpha \beta \mid a b]: a \in\left[a_{\max }\right], b \in\left[b_{\max }\right]\right\}$, is pure$d$-quantum-realizable if there is a state $|\psi\rangle \in \mathbb{C}^{d} \otimes \mathbb{C}^{d}$ and for all $a \in\left[a_{\max }\right]$, there are observables $A_{a}$ on $\mathbb{C}^{d}$ and for all $b \in\left[b_{\max }\right]$, there are observables $B_{b}$ on $\mathbb{C}^{d}$ such that $E[\alpha \beta \mid a b]=\left\langle\psi\left|A_{a} \otimes B_{b}\right| \psi\right\rangle$. A set of joint correlations is $d$-quantum-realizable if it is a probabilistic mixture of pure- $d$-quantum-realizable correlations (this definition accounts for allowing Alice and Bob to share an arbitrary large amount of shared randomness, use POVMs, and share a mixed state). A set of joint correlations is finitely quantum-realizable if there is some $d$ such that the correlations are $d$-quantum-realizable. Note that a set of correlations is local if they are 0-quantum-realizable.

We prove the following theorem.

Theorem 2. For any $d$, there are correlations that are finitely quantum-realizable, but which are not d-quantum-realizable.

We now describe the correlations that we use to prove Theorem 2. Fix some integer $n$. Alice and Bob's possible measurements are parametrized by unit vectors in $\mathbb{R}^{n}, \boldsymbol{a}$ and $\boldsymbol{b}$, respectively. (Note that each party here has an infinite number of possible measurements; we'll reprove the theorem with finite sets of measurements in the next subsection.) The joint correlations are given by

$$
E[\alpha \beta \mid \boldsymbol{a b}]=\boldsymbol{a} \cdot \boldsymbol{b},
$$

where $\boldsymbol{a} \cdot \boldsymbol{b}$ is just the Euclidean inner product of $\boldsymbol{a}$ and $\boldsymbol{b}$. For all $n$, these correlations are finitely quantum-realizable, as the following result shows.

Lemma 5 (Tsirelson [Tsi87]). Let $|\psi\rangle$ be a maximally entangled state on $\mathbb{C}^{d} \otimes \mathbb{C}^{d}$ where $d=2^{\lfloor n / 2\rfloor}$. Then there are two mappings from unit vectors in $\mathbb{R}^{n}$ to observables on $\mathbb{C}^{d}$, one taking $\boldsymbol{a}$ to $A_{\boldsymbol{a}}$, the other taking $\boldsymbol{b}$ to $B_{\boldsymbol{b}}$, such that

$$
\left\langle\psi\left|A_{\boldsymbol{a}} \otimes B_{\boldsymbol{b}}\right| \psi\right\rangle=\boldsymbol{a} \cdot \boldsymbol{b},
$$

for all unit vectors $\boldsymbol{a}, \boldsymbol{b}$.

To show they are not $d$-quantum-realizable, we will use the following characterization. 
Lemma 6 ([Tsi87,AGT06]). Suppose Alice and Bob measure observables $A_{a}$ and $B_{b}$ on a pure quantum state $|\psi\rangle \in \mathbb{C}^{d} \otimes \mathbb{C}^{d}$. Then we can associate a real unit vector $A(a) \in \mathbb{R}^{2 d^{2}}$ with $A_{a}$ (independent of $B_{b}$ ), and a real unit vector $B(b) \in \mathbb{R}^{2 d^{2}}$ with $B_{b}$ (independent of $A_{a}$ ) such that

$$
E[\alpha \beta \mid a b]=\left\langle\psi\left|A_{a} \otimes B_{b}\right| \psi\right\rangle=A(a) \cdot B(b) .
$$

Proof (of Theorem 2). Let $n=2 d^{2}+1$, and consider the joint correlations described in Eq. (34). By Lemma 5, these correlations are finitely quantum-realizable. To show they are not $d$-quantum-realizable, we will show that they lie outside the convex hull of the set of pure- $d$-quantum-realizable correlations.

We do this in the standard way using a Bell inequality, which is a linear function on the vector of correlations, i.e.,

$$
B(E[\alpha \beta \mid \boldsymbol{a b}])=\int \boldsymbol{d a d b} M(\boldsymbol{a}, \boldsymbol{b}) E[\alpha \beta \mid \boldsymbol{a b}],
$$

for some function $M(\boldsymbol{a}, \boldsymbol{b})$, where the integral is over all unit vectors $\boldsymbol{a}, \boldsymbol{b}$ in $\mathbb{R}^{n}$. Here we take $M(\boldsymbol{a}, \boldsymbol{b})=\boldsymbol{a} \cdot \boldsymbol{b}$ so as to apply the results of Sect. 4. Substituting for $E[\alpha \beta \mid \boldsymbol{a} \boldsymbol{b}]$ using Eq. (34), we have

$$
B(E[\alpha \beta \mid \boldsymbol{a b}])=\int \boldsymbol{d a d} \boldsymbol{b}(\boldsymbol{a} \cdot \boldsymbol{b})^{2} .
$$

For any pure- $d$-quantum-realizable correlations, by Lemma 6 there are vectors $A(\boldsymbol{a})$ and $B(\boldsymbol{b})$ in $\mathbb{R}^{2 d^{2}}$, such that the resulting correlations are given by

$$
E[\alpha \beta \mid \boldsymbol{a b}]_{d}=A(\boldsymbol{a}) \cdot B(\boldsymbol{b}) .
$$

Evaluating $B$ on these correlations, we must have

$$
\begin{aligned}
B\left(E[\alpha \beta \mid \boldsymbol{a b}]_{d}\right) & =\int \boldsymbol{d a d} \boldsymbol{b}(\boldsymbol{a} \cdot \boldsymbol{b}) A(\boldsymbol{a}) \cdot B(\boldsymbol{b}) \\
& \leq \max _{A, B} \int \boldsymbol{d a d b}(\boldsymbol{a} \cdot \boldsymbol{b}) A(\boldsymbol{a}) \cdot B(\boldsymbol{b}) \\
& \leq \frac{1}{K_{G}^{\leq}\left(n \mapsto 2 d^{2}\right)} \int \boldsymbol{d a d} \boldsymbol{b}(\boldsymbol{a} \cdot \boldsymbol{b})^{2} \\
& =\frac{1}{K_{G}^{\leq}\left(n \mapsto 2 d^{2}\right)} B(E[\alpha \beta \mid \boldsymbol{a} \boldsymbol{b}]),
\end{aligned}
$$

where $K_{G}^{\leq}\left(n \mapsto 2 d^{2}\right)$ is our lower bound on $K_{G}\left(n \mapsto 2 d^{2}\right)$. Since we chose $n=2 d^{2}+1, K_{G}^{\leq}(n \mapsto n-1)>1$ by Theorem 1 . Hence $B\left(E[\alpha \beta \mid \boldsymbol{a b}]_{d}\right)<B(E[\alpha \beta \mid \boldsymbol{a b}])$ for all pure- $d$-quantum-realizable correlations, which implies that $E[\alpha \beta \mid \boldsymbol{a b}]$ lies outside the convex hull of vectors of the form $E[\alpha \beta \mid \boldsymbol{a b}]_{d}$. We conclude that the correlations in Eq. (34) are not $d$-quantum-realizable.

We conclude this section by noting a slight variant of our result. Suppose we only wish to approximately reproduce some set of quantum correlations, which were originally produced by performing two-outcome measurements on an entangled state with support $\mathbb{C}^{D} \otimes \mathbb{C}^{D}$. Then this can be done with a constant amount of entanglement, where the constant depends on the desired accuracy of the approximation, but not on the dimension $D$ [CHTW09]. In other words, in order to strengthen Theorem 6 to construct correlations that are not approximately $d$-quantum-realizable, we would need to look beyond two-outcome measurements. 
5.1. Reducing the number of questions. A possible objection to the example above is that the number of questions is taken to be infinite. Here we reduce to a finite number of questions in the most straightforward way possible, by discretizing the unit $n$-sphere using an $\varepsilon$-net.

Theorem 3. There are two-party two-outcome correlations with $\exp (\operatorname{poly}(d))$ measurement settings that are finitely quantum-realizable but not d-quantum-realizable.

Proof. To convert the quantum correlations of Eq. (34) into correlations with only a finite number of settings, fix $0<\varepsilon<1$ (to be chosen later) and let $E_{n}^{\varepsilon}$ be an $\varepsilon$-net for $S_{n-1}$ with $(3 / \varepsilon)^{n}$ settings, the existence of which is guaranteed by Lemma 3. Consider the following correlations: Alice's set of possible measurements is $E_{n}^{\varepsilon}$, and so is Bob's (note that we implicitly apply Lemma 5 here). If Alice performs a measurement $\boldsymbol{u} \in E_{n}^{\varepsilon}$ and Bob a measurement $v \in E_{n}^{\mathcal{E}}$, the joint correlation should satisfy

$$
E[\alpha \beta \mid \boldsymbol{u} \boldsymbol{v}]=\boldsymbol{u} \cdot \boldsymbol{v}
$$

just as in our earlier example. These correlations, being a subset of those considered above, are finitely quantum-realizable.

As in the proof of Lemma 2, we use the $\varepsilon$-net to divide the unit sphere into $\left|E_{n}^{\varepsilon}\right|$ regions: For $\boldsymbol{u} \in E_{n}^{\varepsilon}$, let $R_{\boldsymbol{u}}$ be the set of points on $S_{n-1}$ that are closer to $\boldsymbol{u}$ than to any other point in $E_{n}^{\varepsilon}$, and assign points equidistant to two or more points in the net in some arbitrary way. Consider the Bell inequality

$$
B_{\text {finite }}(E[\alpha \beta \mid \boldsymbol{u} \boldsymbol{v}])=\sum_{\boldsymbol{u} \in E_{n}^{\varepsilon}} \int_{\boldsymbol{a} \in R_{\boldsymbol{u}}} \boldsymbol{d a} \sum_{\boldsymbol{v} \in E_{n}^{\varepsilon}} \int_{\boldsymbol{b} \in R_{\boldsymbol{v}}} \boldsymbol{d} \boldsymbol{b}(\boldsymbol{a} \cdot \boldsymbol{b}) E[\alpha \beta \mid \boldsymbol{u} \boldsymbol{v}] .
$$

Evaluating this on the correlations $E[\alpha \beta \mid \boldsymbol{u} \boldsymbol{v}]=\boldsymbol{u} \cdot \boldsymbol{v}$, we obtain

$$
\begin{aligned}
B_{\text {finite }}(E[\alpha \beta \mid \boldsymbol{u} \boldsymbol{v}]) & =\sum_{\boldsymbol{u} \in E_{n}^{\varepsilon}} \int_{\boldsymbol{a} \in R_{\boldsymbol{u}}} \boldsymbol{d} \boldsymbol{a} \sum_{\boldsymbol{v} \in E_{n}^{\varepsilon}} \int_{\boldsymbol{b} \in R_{\boldsymbol{v}}} \boldsymbol{d} \boldsymbol{b}(\boldsymbol{a} \cdot \boldsymbol{b})(\boldsymbol{u} \cdot \boldsymbol{v}) \\
& \geq-2 \varepsilon+\int \boldsymbol{d} \boldsymbol{a} \int \boldsymbol{d} \boldsymbol{b}(\boldsymbol{a} \cdot \boldsymbol{b})^{2} \\
& =B(E[\alpha \beta \mid \boldsymbol{a} \boldsymbol{b}])-2 \varepsilon,
\end{aligned}
$$

where we used

$$
\begin{aligned}
\boldsymbol{u} \cdot \boldsymbol{v} & =\boldsymbol{a} \cdot \boldsymbol{b}+(\boldsymbol{u}-\boldsymbol{a}) \cdot \boldsymbol{b}+\boldsymbol{u} \cdot(\boldsymbol{v}-\boldsymbol{b}) \\
& \geq \boldsymbol{a} \cdot \boldsymbol{b}-\|\boldsymbol{u}-\boldsymbol{a}\|_{2}-\|\boldsymbol{v}-\boldsymbol{b}\|_{2} \\
& \geq \boldsymbol{a} \cdot \boldsymbol{b}-2 \varepsilon
\end{aligned}
$$

and related the value of the Bell inequality with a finite number of settings to the value of our earlier Bell inequality with infinite settings using Eq. (37).

Now consider a pure $d$-dimensional quantum strategy. Let $A(\boldsymbol{u})$ be the $2 d^{2}$ dimensional real unit vector associated with Alice's measurement $\boldsymbol{u}$ and $B(\boldsymbol{v})$ be the vector associated with Bob's measurement $v$ by Lemma 6 and suppose that the resulting correlations are $E[\alpha \beta \mid \boldsymbol{u v}]_{d}$. We need to show that $B_{\text {finite }}\left(E[\alpha \beta \mid \boldsymbol{u} \boldsymbol{v}]_{d}\right)$ is strictly smaller than $B_{\text {finite }}(E[\alpha \beta \mid \boldsymbol{u} \boldsymbol{v}])$. We'll do this by relating $B_{\text {finite }}\left(E[\alpha \beta \mid \boldsymbol{u} \boldsymbol{v}]_{d}\right)$ to $B(E[\alpha \beta \mid \boldsymbol{a b}])$ and applying the inequality proved in Eq. (42). 
The finite strategy (i.e., the mappings $A(\boldsymbol{u})$ and $B(\boldsymbol{v}))$ induces a strategy for the correlations where we had an infinite number of questions. Recall that a strategy in the infinite case was equivalent to a mapping from $S_{n-1}$ to unit vectors in $\mathbb{R}^{2 d^{2}}$ by Lemma 6 . Consider the mapping defined as follows: First map $\boldsymbol{a}$ to the closest point $\boldsymbol{u}$ in the $\varepsilon$-net, then to the vector $A(\boldsymbol{u})$, and similarly for Bob's strategy. For this strategy:

$$
\begin{aligned}
B\left(E[\alpha \beta \mid \boldsymbol{a} \boldsymbol{b}]_{d}\right) & =\sum_{\boldsymbol{u} \in E_{n}^{\varepsilon}} \int_{\boldsymbol{a} \in R_{\boldsymbol{u}}} \boldsymbol{d a} \sum_{\boldsymbol{v} \in E_{n}^{\varepsilon}} \int_{\boldsymbol{b} \in R_{\boldsymbol{v}}} \boldsymbol{d} \boldsymbol{b}(\boldsymbol{a} \cdot \boldsymbol{b}) A(\boldsymbol{u}) \cdot B(\boldsymbol{v}) \\
& =\sum_{\boldsymbol{u} \in E_{n}^{\varepsilon}} \int_{\boldsymbol{a} \in R_{\boldsymbol{u}}} \boldsymbol{d a} \sum_{\boldsymbol{v} \in E_{n}^{\varepsilon}} \int_{\boldsymbol{b} \in R_{\boldsymbol{v}}} \boldsymbol{d} \boldsymbol{b}(\boldsymbol{a} \cdot \boldsymbol{b}) E[\alpha \beta \mid \boldsymbol{u} \boldsymbol{v}] \\
& =B_{\mathrm{finite}}\left(E[\alpha \beta \mid \boldsymbol{u} \boldsymbol{v}]_{d}\right) .
\end{aligned}
$$

Combining the above calculations with Eq. (42), we calculate

$$
\begin{aligned}
B_{\text {finite }}\left(E[\alpha \beta \mid \boldsymbol{u} \boldsymbol{v}]_{d}\right) & =B\left(E[\alpha \beta \mid \boldsymbol{a b}]_{d}\right) \\
& \leq \frac{1}{K_{G}^{\leq}\left(n \mapsto 2 d^{2}\right)} B(E[\alpha \beta \mid \boldsymbol{a} \boldsymbol{b}]) .
\end{aligned}
$$

Take $n=2 d^{2}+2$ (for ease of calculation, so that the $\Gamma$-functions cancel), and we have

$$
K_{G}^{\leq}\left(2 d^{2}+2 \mapsto 2 d^{2}\right)=1+\Delta_{d},
$$

where $\Delta_{d}=1 /\left(4 d^{2}\left(d^{2}+1\right)\right)$.

Now we can put everything together. Starting with Eq. (55), we have

$$
\begin{aligned}
B_{\text {finite }}\left(E[\alpha \beta \mid \boldsymbol{u} \boldsymbol{v}]_{d}\right) & \leq \frac{1}{1+\Delta_{d}} B(E[\alpha \beta \mid \boldsymbol{a b}]) \\
& <\left(1-\frac{\Delta_{d}}{2}\right) B(E[\alpha \beta \mid \boldsymbol{a b}]) \\
& =B(E[\alpha \beta \mid \boldsymbol{a b}])-\frac{\Delta_{d}}{2 n}
\end{aligned}
$$

where (58) is valid because $0<\Delta_{d}<1$, and in Eq. (59) we substituted

$$
B(E[\alpha \beta \mid \boldsymbol{a b}])=\int \boldsymbol{d} \boldsymbol{a} \int \boldsymbol{d} \boldsymbol{b}(\boldsymbol{a} \cdot \boldsymbol{b})^{2}=\frac{1}{n} Y_{n}^{2}=\frac{1}{n},
$$

as calculated in Sect. 4 .

Thus, so long as we choose $2 \varepsilon \leq \Delta_{d} /(2 n)$, we can combine Eqs. (59) and (47) to obtain

$B_{\text {finite }}\left(E[\alpha \beta \mid \boldsymbol{u} \boldsymbol{v}]_{d}\right)<B(E[\alpha \beta \mid \boldsymbol{a b}])-\frac{\Delta_{d}}{2 n} \leq B(E[\alpha \beta \mid \boldsymbol{a b}])-2 \varepsilon \leq B_{\text {finite }}(E[\alpha \beta \mid \boldsymbol{u} \boldsymbol{v}])$,

i.e., we have proved that $B_{\text {finite }}\left(E[\alpha \beta \mid \boldsymbol{u v}]_{d}\right)<B_{\text {finite }}(E[\alpha \beta \mid \boldsymbol{u v}])$ meaning that our correlations are not $d$-quantum-realizable. The number of measurement settings is

$$
(3 / \varepsilon)^{n}=\left(12 n / \Delta_{d}\right)^{n}=\exp \left(O\left(d^{2} \log d\right)\right) .
$$




\section{Proof of Equality in Lemma 1}

The fact that equality holds in the inequality in Eq. (5) of Lemma 1 follows directly from the following proposition.

Proposition 1. For all positive integers $r$, for all $r \times r$ real-valued matrices $M_{i j}$, and any $\epsilon>0$, there exists a measurable function $M^{\prime}: S_{n-1} \times S_{n-1} \rightarrow \mathbb{R}$ with $\int \boldsymbol{d a d} \boldsymbol{b}\left|M^{\prime}(\boldsymbol{a}, \boldsymbol{b})\right|=1$, such that

$$
\frac{\int \boldsymbol{d} \boldsymbol{a} \boldsymbol{d} \boldsymbol{b} M^{\prime}(\boldsymbol{a}, \boldsymbol{b}) \boldsymbol{a} \cdot \boldsymbol{b}}{\max _{A, B: S_{n-1} \rightarrow S_{m-1} \int \boldsymbol{d} \boldsymbol{a} \boldsymbol{d} \boldsymbol{b} M^{\prime}(a, b) A(a) \cdot B(b)}} \geq \frac{\max _{\left(\boldsymbol{a}_{i}, \boldsymbol{b}_{j}\right)} \sum_{i, j} M_{i j} \boldsymbol{a}_{i} \cdot \boldsymbol{b}_{j}}{\max _{\left(\boldsymbol{a}_{i}^{\prime}, \boldsymbol{b}_{j}^{\prime}\right)} \sum_{i, j} M_{i j} \boldsymbol{a}_{i}^{\prime} \cdot \boldsymbol{b}_{j}^{\prime}}-\epsilon .
$$

Proof. Let $f, g:[r] \rightarrow S_{n-1}$ and $f^{\prime}, g^{\prime}:[r] \rightarrow S_{m-1}$ be vector valued functions, and let $f^{*}$ and $g^{*}$ be such that they give a sequence $\left(f^{*}(i), g^{*}(j)\right)_{i, j=1}^{r}=\left(\boldsymbol{a}_{i}^{*}, \boldsymbol{b}_{j}^{*}\right)_{i=1}^{r}$ that maximizes $\sum_{i, j} M_{i j} \boldsymbol{a}_{i} \cdot \boldsymbol{b}_{j}$. Set

$$
M^{\prime}(\boldsymbol{a}, \boldsymbol{b})=\sum_{i, j} M_{i j} \delta\left(\boldsymbol{a}-\boldsymbol{a}_{i}^{*}\right) \delta\left(\boldsymbol{b}-\boldsymbol{b}_{j}^{*}\right)
$$

where $\delta(\cdot)$ denotes the Dirac delta function. This causes the numerators of (63) to be equal. For the denominator of the left-hand side of (63), we have

$$
\begin{aligned}
& \quad \max _{A, B: S_{n-1} \rightarrow S_{m-1}} \int d \boldsymbol{a d b} M^{\prime}(\boldsymbol{a}, \boldsymbol{b}) A(\boldsymbol{a}) \cdot B(\boldsymbol{b}) \\
& =\max _{A^{\prime}, B^{\prime}:\left(f^{*}(i), g^{*}(j)\right) \rightarrow S_{m-1}} \sum_{i, j} M_{i j} A^{\prime}\left(f^{*}(i)\right) \cdot B^{\prime}\left(g^{*}(j)\right) \\
& \leq \max _{f^{\prime}, g^{\prime}} \sum_{i, j} M_{i j} f^{\prime}(i) \cdot g^{\prime}(j),
\end{aligned}
$$

where the inequality follows because the second maximization is over a subset of the set that the last equation is maximized over. The problem that the delta function is a distribution and not really a function may be remedied by considering an approximation to it sufficient to obtain the inequality to within $\epsilon$. All that remains is to rescale $M^{\prime}(\boldsymbol{a}, \boldsymbol{b})$ so that it satisfies $\int \boldsymbol{d} \boldsymbol{a d} \boldsymbol{b}\left|M^{\prime}(\boldsymbol{a}, \boldsymbol{b})\right|=1$. This gives the result.

\section{Proof that the Bound on $K_{G}(n \mapsto m)$ is Nontrivial}

Here we establish the following lemma.

Lemma 7. The function

$$
f(n)=\frac{1}{\sqrt{n}} \frac{\Gamma\left(\frac{n+1}{2}\right)}{\Gamma\left(\frac{n}{2}\right)}
$$

is strictly increasing on integers $n=1,2, \ldots$. 
Proof. For $n \leq 9$, just evaluate $f(n)$. For $n>9$, we use the following bound on $\log \Gamma(x)$, first proved by Robbins [Rob55] for integer values of $x$, but which Matsunawa observed [Mat76, Remark 4.1] is also valid for real values of $x \geq 2$ :

$$
\sqrt{2 \pi} x^{x+1 / 2} e^{-x+1 /(12 x+1)}<\Gamma(x+1)<\sqrt{2 \pi} x^{x+1 / 2} e^{-x+1 /(12 x)} .
$$

Using this bound, we obtain

$$
\begin{aligned}
\log \frac{f(n+1)}{f(n)}= & -\frac{1}{2} \log \left(1+\frac{1}{n}\right)+\log \frac{n}{2}+2 \log \Gamma\left(\frac{n}{2}\right)-2 \log \Gamma\left(\frac{n+1}{2}\right) \\
\geq & -\frac{1}{2} \log \left(1+\frac{1}{n}\right)+\log \left(1+\frac{1}{n / 2-1}\right)-n \log \left(1+\frac{1}{n-2}\right) \\
& +\frac{2}{6 n-11}-\frac{2}{6 n-6} .
\end{aligned}
$$

Now use

$$
\frac{1}{n}-\frac{1}{2 n^{2}}+\frac{1}{3 n^{3}}-\frac{1}{4 n^{4}} \leq \log \left(1+\frac{1}{n}\right) \leq \frac{1}{n}-\frac{1}{2 n^{2}}+\frac{1}{3 n^{3}},
$$

(which is valid for all $n \geq 1$ ), and we obtain

$$
\begin{aligned}
\log \frac{f(m+10)}{f(m+9)} \\
\geq\left(14 m^{7}+679 m^{6}+13923 m^{5}+155346 m^{4}+1005620 m^{3}\right. \\
\left.\quad+3684139 m^{2}+6679947 m+3828140\right) /\left(12(m+7)^{4}(m+8)(m+9)^{3}(6 m+43)\right),
\end{aligned}
$$

which is obviously positive when $m \geq 0$, i.e., when $n \geq 9$. Thus $f(n)$ is strictly increasing.

Acknowledgements. We thank Ronald de Wolf for discussions about the Hidden Matching problem and we thank Vid Stojevic for pointing out a problem with an earlier version of Lemma 1.

Open Access This article is distributed under the terms of the Creative Commons Attribution Noncommercial License which permits any noncommercial use, distribution, and reproduction in any medium, provided the original author(s) and source are credited.

\section{References}

[ADR82] Aspect, A., Dalibard, J., Roger, G.: Experimental test of bell's inequalities using time- varying analyzers. Phys. Rev. Lett. 49(25), 1804-1807 (1982)

[AGR81] Aspect, A., Grangier, P., Roger, G.: Experimental tests of realistic local theories via bell's theorem. Phys. Rev. Lett. 47(7), 460-463 (1981)

[AGR82] Aspect, A., Grangier, P., Roger, G.: Experimental realization of einstein-podolsky-rosen-bohm gedankenexperiment: a new violation of bell's inequalities. Phys. Rev. Lett. 49(2), 91-94 (1982)

[AGT06] Acín, A., Gisin, N., Toner, B.: Grothendieck's constant and local models for noisy entangled quantum states. Phys. Rev. A 73(6), 062105 (2006)

[AMMN06] Alon, N., Makarychev, K., Makarychev, Y., Naor, A.: Quadratic forms on graphs. Invent. Math. 163(3), 499-522 (2006)

[AN04] Alon, N., Naor, A.: Approximating the cut-norm via Grothendieck's inequality. In: Proc. of the 36th ACM STOC, New York: ACM Press, 2004, pp. 72-80 
[BBLV09] Briët, J., Buhrman, H., Lee, T., Vidick, T.: Multiplayer XOR games and quantum communication complexity with clique-wise entanglement. http://arxiv.org/abs/0911.4007v1 [quant.ph], 2009

[Bel64] Bell, J.S.: On the einstein-podolsky-rosen paradox. Physics 1, 195-200 (1964)

[BOFV10a] Briët, J., Oliveira Filho, F.d., Vallentin, F.: Grothendieck inequalities for semidefinite programs with rank constraint. http://arxiv.org/abs/1011.1754v1 [math.OC], 2010

[BOFV10b] Briët, J., Oliveira Filho, F.d., Vallentin, F.: The positive semidefinite grothendieck problem with rank constraint. In: S. Abramsky, C. Gavoille, C. Kirchner, F. Meyer auf der Heide, P. Spirakis (eds.) Automata, Languages and Programming, Volume $\mathbf{6 1 9 8}$ of Lecture Notes in Computer Science, Berlin/Heidelberg: Springer, 2010, pp. 31-42

$\left[\mathrm{BPA}^{+} 08\right] \quad$ Brunner, N., Pironio, S., Acin, A., Gisin, N., Méthot, A.A., Scarani, V.: Testing the dimension of hilbert spaces. Phys. Rev. Lett. 100(21), 210503 (2008)

[BRSW10] Buhrman, H., Regev, O., Scarpa, G., Wolf, R.d.: Near-Optimal and Explicit Bell Inequality Violations. http://arxiv.org/abs/1012.5043v2 [quant.ph], 2010, to appearin proc. IEEE Complexity 2011

[BYJK08] Bar-Yossef, Z., Jayram, T.S., Kerenidis, I.: Exponential separation of quantum and classical one-way communication complexity. SIAM J. Comput. 38(1), 366-384 (2008)

[CHSH69] Clauser, J.F., Horne, M.A., Shimony, A., Holt, R.A.: Proposed experiment to test local hidden-variable theories. Phys. Rev. Lett. 23, 880-884 (1969)

[CHTW04] Cleve, R., Høyer, P., Toner, B., Watrous, J.: Consequences and limits of nonlocal strategies. In: Proceedings of the 19th IEEE Conference on Computational Complexity (CCC 2004), Piscataway, NJ: IEEE, 2004, pp. 236-249

[CHTW09] Cleve, R., Høyer, P., Toner, B., Watrous, J.: 2009 Journal version of [CHTW04], in preparation available at http://arxiv.org/abs/quant.ph/0404079v2, 2010

[CSUU08] Cleve, R., Slofstra, W., Unger, F., Upadhyay, S.: Perfect parallel repetition theorem for quantum xor proof systems. Computational Complexity 17(2), 282-299 (2008)

[CW04] Charikar, M., Wirth, A.: Maximizing quadratic programs: Extending grothendieck's inequality. In: FOCS '04: Proceedings of the 45th Annual IEEE Symposium on Foundations of Computer Science, Washington, DC: IEEE Computer Society, 2004, pp. 54-60

[Dav84] Davie, A.M.: Lower bound for $K_{G}, 1984$ Unpublished note

[FR94] Fishburn, P.C., Reeds, J.A.: Bell inequalities, grothendieck's constant, and root two. SIAM J. Discrete Math. 7(1), 48-56 (1994)

[GKP94] Graham, R.L., Knuth, D.E., Patashnik, O.: Concrete Mathematics: A Foundation for Computer Science second edition. Reading MA: Addison-Wesley, 1994

[Gro53] Grothendieck, A.: Résumé de la théorie métrique des produits tensoriels topologiques. Boletim Da Sociedade de Matemática de S ao Paulo 8, 1 (1953)

[JPPG $\left.{ }^{+} 10\right]$ Junge, M., Palazuelos, C., Prez-Garca, D., Villanueva, I., Wolf, M.: Unbounded violations of bipartite bell inequalities via operator space theory. Commun. Math. Phys. 1-25 (2010)

[Kri79] Krivine, J.L.: Constantes de grothendieck et fonctions de type positif sur les sphères. Adv. Math. 31, 16-30 (1979)

[LMSS08] Linial, N., Mendelson, S., Schechtman, G., Shraibman, A.: Complexity measures of sign matrices. Combinatorica 27(4), 439-463 (2008)

[LS07] Linial, N., Shraibman, A.: Lower bounds in communication complexity based on factorization norms. In: STOC '07: Proceedings of the thirty-ninth annual ACM symposium on Theory of computing, New York: ACM, 2007, pp. 699-708

[Mat76] Matsunawa, T.: Some inequalities based on inverse factorial series. Ann. Inst. Statist. Math. 28(1), 291-305 (1976)

[NC00] Nielsen, M.A., Chuang, I.L.: Quantum Computation and Quantum Information. New York: Cambridge University Press, 2000

[Nes98] Nesterov, Y.E.: Semidefinite relaxations and nonconvex quadratic optimization. Optimization Methods and Software (9), 141-160 (1998)

[PGWP ${ }^{+}$08] Pérez-García, D., Wolf, M.M., Palazuelos, C., Villanueva, I., Junge, M.: Unbounded violation of tripartite bell inequalities. Commun. Math. Phys. 279, 455-486 (2008)

[Pis99] Pisier, G.: The volume of convex bodies and Banach space geometry. Cambridge: Cambridge Univ Pr, 1999

[PV08] Pál, K.F., Vértesi, T.: Efficiency of higher-dimensional hilbert spaces for the violation of bell inequalities. Phys. Rev. A 77(4), 042105 (2008)

[PV09] Pál, K., Vértesi, T.: Quantum bounds on bell inequalities. Phys. Rev. A 79(2), 022120 (2009)

[Ree91] Reeds, J.A.: A new lower bound on the real Grothendieck constant, 1991. Unpublished note, available at http://www.dtc.umn.edu/reedsj/bound2.dvi

[Rie74] Rietz, R.: A proof of the grothendieck inequality. Israel J. Math. 19(3), 271-276 (1974) 
$\left[\mathrm{RKM}^{+}\right.$01] Rowe, M.A., Kielpinski, D., Meyer, V., Sackett, C.A., Itano, W.M., Monroe, C., Wineland, D.J.: Experimental violation of a bell's inequality with efficient detection. Nature 409, 791794 (2001)

[Rob55] Robbins, H.: A remark of stirling's formula. Amer. Math. Monthly 62(1), 26-29 (1955)

[RS09] Raghavendra, P., Steurer, D.: Towards computing the Grothendieck constant. In: Proceedings of the twentieth Annual ACM-SIAM Symposium on Discrete Algorithms, Philadelphia: Society for Industrial and Applied Mathematics, 2009, pp. 525-534

[RT07] Regev, O., Toner, B.: Simulating quantum correlations with finite communication. In: Proceedings of the 48th Annual IEEE Symposium on Foundations of Computer Science (FOCS 2007), Washington, DC: IEEE Computer Society, 2007, pp. 384-394

[TBZG98] Tittel, W., Brendel, J., Zbinden, H., Gisin, N.: Violation of bell inequalities by photons more than $10 \mathrm{~km}$ apart. Phys. Rev. Lett. 81(17), 3563-3566 (1998)

[Tsi87] Tsirelson, B.S.: Quantum analogues of the bell inequalities. The Case of Two Spatially Separated Domains. J. Soviet Math. 36, 557-570 (1987)

[VP09] Vértesi, T., Pál, K.: Bounding the dimension of bipartite quantum systems. Phys. Rev. A 79(4), 042106 (2009)

[WCD08] Wehner, S., Christandl, M., Doherty, A.: Lower bound on the dimension of a quantum system given measured data. Phys. Rev. A 78(6), 062112 (2008)

Communicated by M.B. Ruskai 